\title{
RESEARCH ON FOREST FLAME RECOGNITION ALGORITHM BASED ON IMAGE FEATURE
}

\author{
Zuoning Wang, Pengfei Liu*, Tiejun Cui' \\ School of Urban and Environmental Sciences, Tianjin Normal University, liupengfei0920@163.com
}

KEY WORDS: Forest Fire, Image Processing, Feature Analysis, Color Model, Cluster Analysis, Flame Recognition

\begin{abstract}
:
In recent years, fire recognition based on image features has become a hotspot in fire monitoring. However, due to the complexity of forest environment, the accuracy of forest fireworks recognition based on image features is low. Based on this, this paper proposes a feature extraction algorithm based on $\mathrm{YCrCb}$ color space and $\mathrm{K}$-means clustering. Firstly, the paper prepares and analyzes the color characteristics of a large number of forest fire image samples. Using the K-means clustering algorithm, the forest flame model is obtained by comparing the two commonly used color spaces, and the suspected flame area is discriminated and extracted. The experimental results show that the extraction accuracy of flame area based on $\mathrm{YCrCb}$ color model is higher than that of HSI color model, which can be applied in different scene forest fire identification, and it is feasible in practice.
\end{abstract}

\section{INTRODUCTION}

Forest fire sudden strong, dangerous, is a more difficult to deal with the rescue disaster.Forest fires are often developed rapidly, harmed, difficult to fight, difficult to be found, so the timely and accurate detection of fire for the early extinguish the fire, reduce economic losses is of great significance.

In recent years, fire recognition based on image features has become a hotspot in fire monitoring research. Image-based methods to monitor forest fires can make up for the shortcomings of past forest fire monitoring. Although the video fire detection technology based on video images is compared with the traditional wood fire detection technology, it has a wide range of detection, anti-interference, wide application range, low false alarm rate, strong response, high sensitivity, low cost of information and But also in the current detection accuracy, testing stability, etc., still a lot of shortcomings.

Yan et al. ${ }^{[1]}$ using the Otsu algorithm to segment the image, extract the suspected flame area color distribution characteristics and shape characteristics, and through the fuzzy neural network to obtain the test results. Zhu et al ${ }^{[2]}$ etc. to 4 followed forest flame image as a sample, on the basis of RGB color space, the color, area and edge of the forest flame were analyzed, and then the flame area was extracted; Liang et al. ${ }^{[3]}$ The paper chooses a segmentation image of $\mathrm{H}, \mathrm{S}$ and I components in the HSI color model, and then proposes a forest fire image segmentation method based on HSI model and regional growth. $\mathrm{Yu}^{[4]}$ HSI space based on the color feature clustering, obtained the suspected flame segmentation image, through the extraction of the flame color and shape characteristics, constitute the flame feature vector, and to achieve a BP neural network model based on the fire flame image recognition algorithm.

Based on the image feature processing technology, this paper improves the accuracy of forest fire image recognition by optimizing the existing image flame recognition algorithm, and maximizes the influence factors of forest flame recognition to achieve the early and accurate fireworks identification effect. And to study the forest flame detection algorithm based on image feature such as forest, in order to provide an effective and feasible new method for forest flame identification algorithm to reduce or avoid the adverse effects of forest fires.

\section{GENERAL IDEA}

The forest flame model is a mathematical model which can judge whether there is a flame in the forest image. In this paper, the forest fire image is extracted and extracted according to the color image reading, color space conversion, $\mathrm{k}$ mean clustering, chroma component acquisition and threshold segmentation (Figure 1),

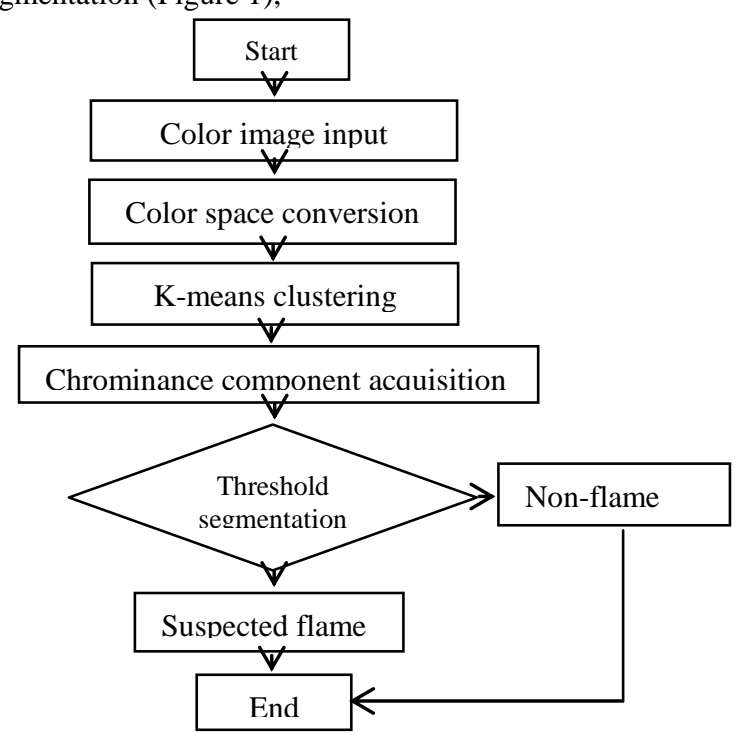

Figure 1 Forest Flame Segmentation Extraction Flow Chart

\subsection{Color space conversion}

The color feature is a global feature based on the pixel, which describes the surface properties of the image corresponding to the image or image area. The selection of the appropriate color

\footnotetext{
* Corresponding author
} 
model is of great significance for the extraction of flame. By comparing the effects of two commonly used HSI and $\mathrm{YCrCb}$ color models in flame image segmentation and extraction, this paper chooses a better color model for flame segmentation..

\subsection{HSI color model}

The HSI color model is based on the human's sense of color, and uses Hue, Saturation, and Intensity to describe the color, which is more suitable for the human visual system than the RGB color model. The formula from the RGB color model to the HSI color model is as follows:

$H=\left\{\begin{array}{ll}\theta, & G \geq B \\ 2 \pi-\theta, & G<B\end{array}\right.$,

among them: $\theta=\cos ^{-1}\left(\frac{(R-G)+(R-B)}{2 \sqrt{(R-G)^{2}+(R-B)(G-B)}}\right)$

$S=1-3[\min (R, G, B)] /(R+G+B)$

$I=(R+G+B) / 3$

The brightness I corresponds to the imaging brightness and image gray level, regardless of the color information of the image, there is no obvious feature on the image, so according to the $\mathrm{H}$ and I components can not be on the forest flame. The saturation of the image is the purity of the color ${ }^{[5]}$, the flame area is high saturation, and the sky area is low saturation, so the $\mathrm{S}$ component can exclude the sky, but can not exclude the saturation of the relatively high green trees, such as Figure 2 shows. HSI color model can better distinguish the color, saturation, brightness, the use of HSI model of forest flame segmentation effect is poor.

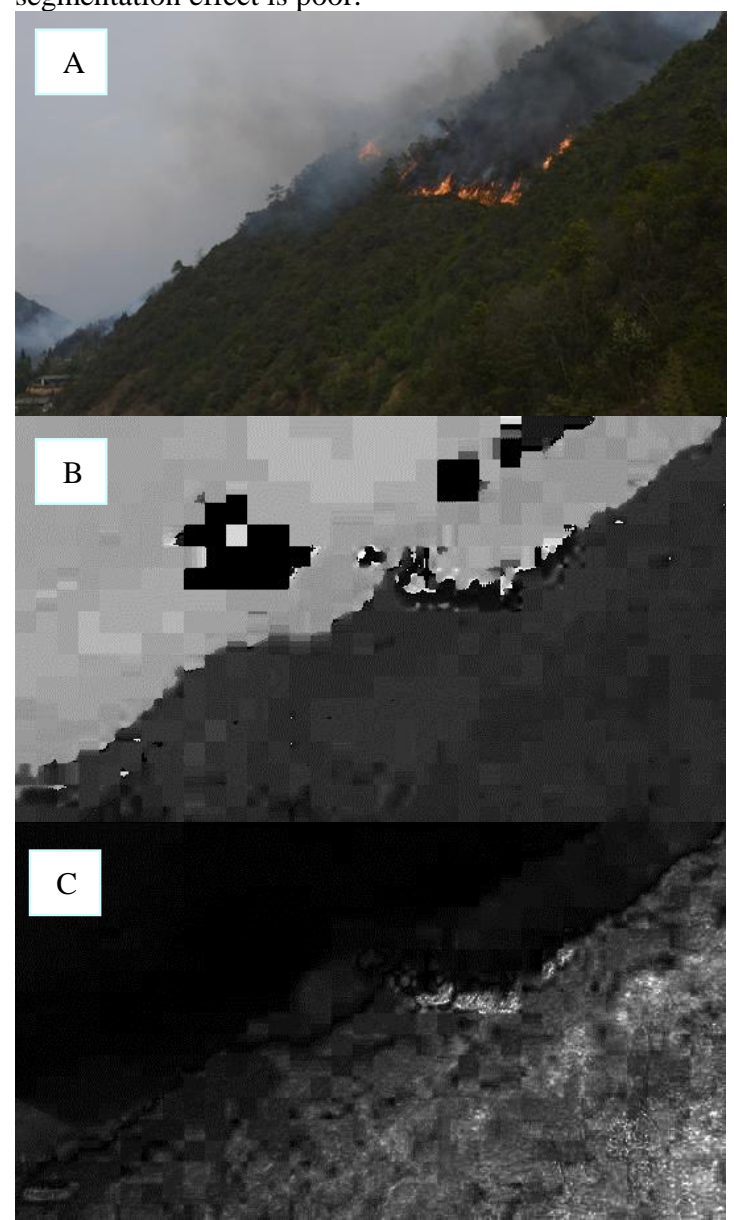

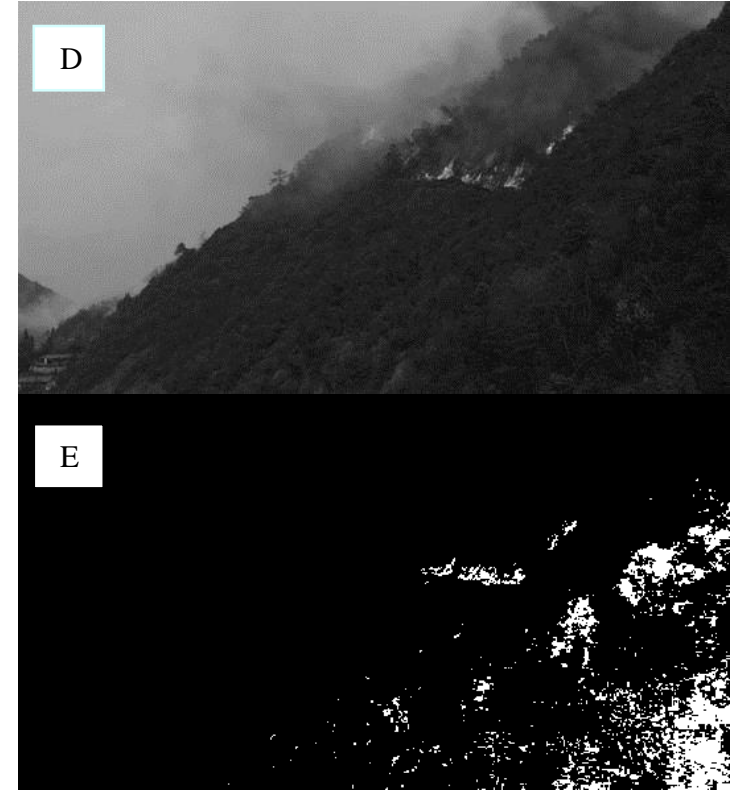

Figure 2. Color Space Conversion Based on HSI Color Model ( A: forest flame original image; $\mathrm{B}$ : $\mathrm{H}$ component; $\mathrm{C}$ : $\mathrm{S}$ component; D: I component; E: the forest flame segmentation results)

\subsection{YCbCr color model}

$\mathrm{Y}$ is the luminance component, $\mathrm{Cr}$ and $\mathrm{Cb}$ together represent the color of the color information, $\mathrm{Cr}$ is the red color component, $\mathrm{Cb}$ is the blue chrominance component; $\mathrm{YCrCb}$ has a color brightness and two represent the color difference signal, and RGB only represents the color of the red, Green and blue. RGB color model to $\mathrm{YCrCb}$ color model formula is as follows:

$$
\begin{aligned}
& Y=0.299 \times R+0.587 \times G+0.114 \times B ; \\
& C b=0.5-0.168736 \times R-0.331264 \times G+0.5 \times B ; \\
& C r=0.5+0.5 \times R-0.418688 \times G-0.081312 \times B ;
\end{aligned}
$$

As the color of the flame is yellow and red, the $\mathrm{Y}$ component can not achieve the division of the forest flame, $\mathrm{Cr}$ is the difference between the red component and the brightness, the channel, the flame area is darker than other regions, the forest flame split effect is better, After the K-means clustering, the flame image is more clear, more obvious features. $\mathrm{Cb}$ is the difference between the blue component and the brightness, in the forest flame segmentation also has some effect.

The results show that the flame value of the forest flame in the $\mathrm{Cr}$ component is greater than 140 can better segment the flame area and other areas; However, the flame image there are two common noise, yellow exposed soil (Cr component in the 140155 ) And orange sunset (Cr component between 170-195), so the yellow exposed soil and sunset are also mistaken for the flame area, but according to the flame of the movement characteristics and shape characteristics can be excluded yellow exposed soil, sunset and other noise interference (Figure 3). 


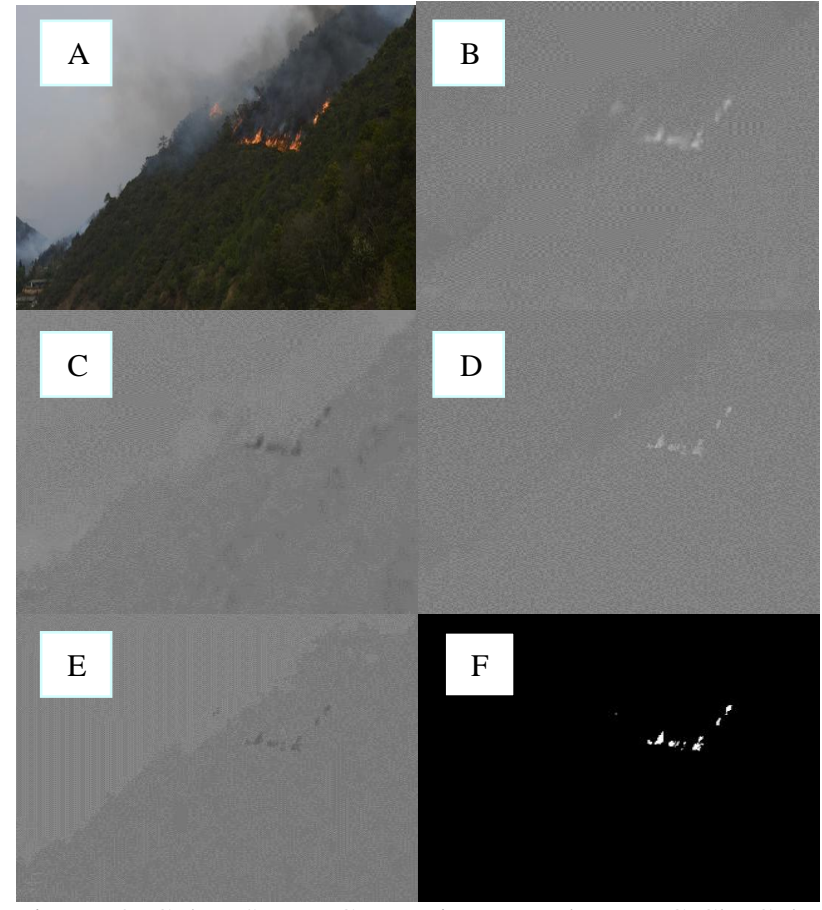

Figure 3 Color Space Conversion Based on $\mathrm{YCrCb}$ Color Model (A: forest flame original image; $\mathrm{B}$ : $\mathrm{Cr}$ component; $\mathrm{C}$ : $\mathrm{Cb}$ component; D: K-means value after the $\mathrm{Cr}$ component; $\mathrm{E}: \mathrm{K}$ -means value after the $\mathrm{Cb}$ component; $\mathrm{F}$ : $\mathrm{Cr}$ component binarization )

\section{$1.4 \mathrm{~K}$ - means clustering method}

K-means method has a good effect on the segmentation of forest flame image. It also has the advantage that other clustering algorithms can not match in dealing with a large number of data sets. However, since K-means clustering minimizes the objective function as the condition of clustering, And the initial clustering center is randomly selected, it is easier to fall into the local optimum, resulting in $\mathrm{K}$-means sensitivity to the initial clustering center, and different initial clustering centers may lead to completely different clustering effects ${ }^{[7]}$. Therefore, according to the particularity of the forest environment, this paper makes a lot of experiments on the initial clustering center of the K-means algorithm, and then determines the initial clustering $\mathrm{K}$ value applied to the forest flame identification, and overcomes the above defects and more accurately divides the forest flame area. The K-means clustering is performed when the $K$ values are set to 5 and 8 respectively. Experiments show that $\mathrm{K}$ take 8, the flame of the split better,the results are shown in Figure 4:

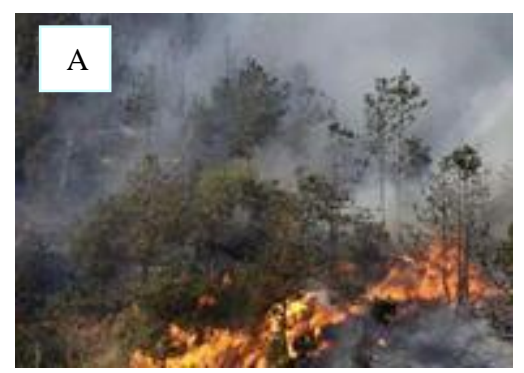

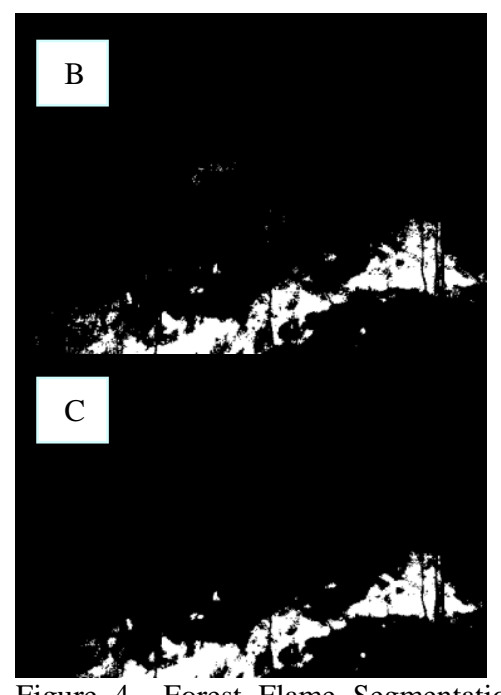

Figure 4 Forest Flame Segmentation Results Based on KMeans Clustering Method(A: forest flame original image; $\mathrm{B}, \mathrm{C}$ :the result of the division of forest flames for $\mathrm{K} 5$ and 8)

\section{EXPERIMENT ANALYSIS}

The experimental samples of this paper are divided into two parts: (1), forest fireworks positive samples; (2), forest nonflame negative samples. The sample selection follows the following principles: (1), the positive sample through the acquisition of forest video surveillance screenshots to obtain a large number of forest fire early flame images; (2), the positive sample to ensure the objectivity at the same time, as much as possible to collect forest fires early seasons, different time periods, different weather conditions and different forest tree species and other environmental flame images to ensure that the richness of the sample and algorithm. (3), negative samples to take full account of the diversity of sources of fireworks and uncertainties, thereby reducing miscarriage of justice.

The K-means clustering of the converted HSI and $\mathrm{YCrCb}$ images is performed first, and the initial number of clusters $\mathrm{K}$ is chosen as 8. Secondly, the HSI and $\mathrm{YCrCb}$ color models after the K-means clustering are separated by the split method in the Opencv library.

The K-means clustering of the converted HSI and $\mathrm{YCrCb}$ images is performed first, and the initial number of clusters $\mathrm{K}$ is chosen as 8. Secondly, the HSI and YCrCb color models after the K-means clustering are separated by the split method in the Opencv library. The S-component and the $\mathrm{Cr}$ component are used to divide the threshold of the flame area. When the $\mathrm{S}$ component is used to divide the gray value, the gray threshold is selected as 105 , and when the Cr component is used, the gray threshold is selected as 140, and the pixel point larger than the threshold is defined as Flame area, and then extract the binarized forest flame image.

Figure 5 shows the results of segmentation and extraction of forest flames using $\mathrm{S}$ and $\mathrm{Cr}$ components, respectively. In the experiment, both methods can separate the flame image. However, in the flame image segmented by the S component, the noise is large, and the difficulty of the later generation of the flame image is increased 


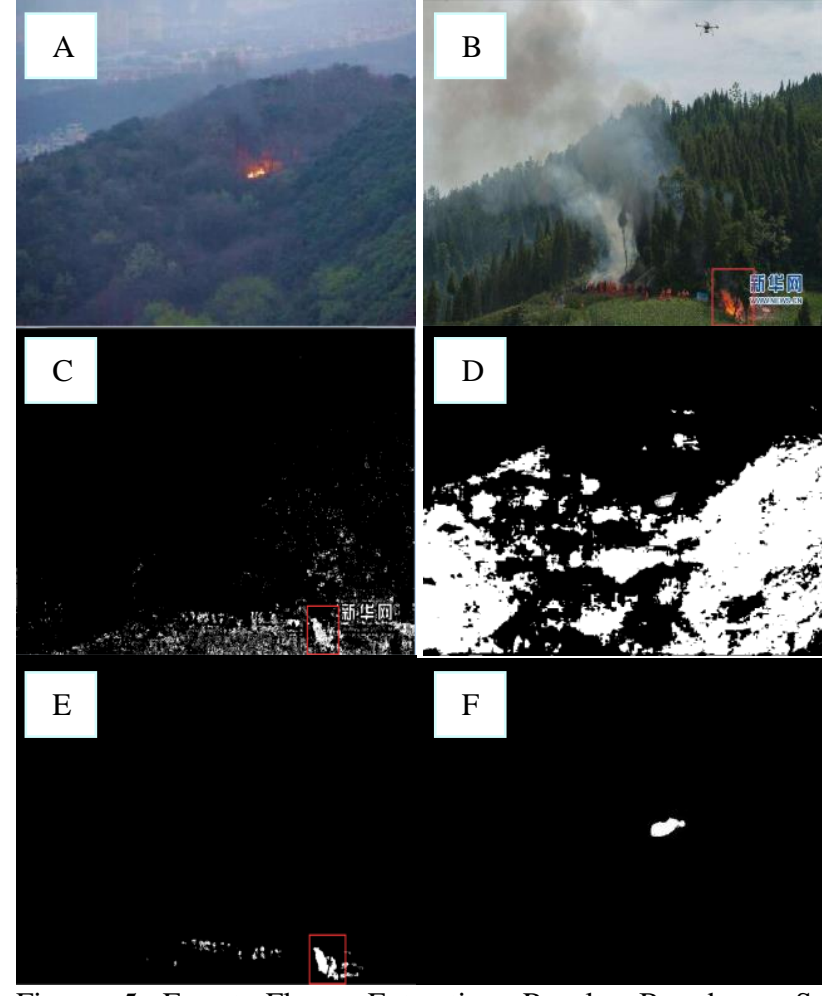

Figure 5 Forest Flame Extraction Results Based on SComponent, Cr Component and K-means Clustering(A,B:the original image of the forest flame, C,D:the $\mathrm{S}$ component segmentation result, E:Cr component segmentation result)

Table 1. Forest Flame Sample Data Processing Results

\begin{tabular}{|c|c|c|c|}
\hline $\begin{array}{l}\text { Number } \\
\text { of } \\
\text { samples }\end{array}$ & $\begin{array}{c}\text { Flame } \\
\text { Segmentation } \\
\text { Accuracy }\end{array}$ & $\begin{array}{c}\text { Accuracy } \\
(\%)\end{array}$ & Sample description \\
\hline 50 & 48 & 96 & $\begin{array}{l}\text { Large area of forest } \\
\text { small flames }\end{array}$ \\
\hline 50 & 50 & 100 & The larger flames \\
\hline 11 & 10 & 90.9 & Dark green trees \\
\hline 8 & 7 & 87.5 & Yellow grass \\
\hline 20 & 19 & 95 & Summer \\
\hline 4 & 4 & 100 & fireFall forest fire \\
\hline 5 & 5 & 100 & Winter forest fire \\
\hline 18 & 17 & 94.4 & Sunny forest fire \\
\hline 21 & 20 & 95.2 & Cloudy forest fireThe \\
\hline 3 & 3 & 100 & $\begin{array}{l}\text { forest is bare yellow } \\
\text { land }\end{array}$ \\
\hline 6 & 5 & 83.3 & Sunset \\
\hline 9 & 8 & 88.9 & sun \\
\hline 12 & 11 & 91.7 & Forest fauna \\
\hline
\end{tabular}

By experimenting with 150 samples, when the initial clustering center is 5 , the flame can not be segmented accurately when the k-means is used for secondary division. After repeated experiments, when the initial cluster center is 8 , the flame recognition effect is better. Compared with the HSI color model, based on the $\mathrm{YCrCb}$ color model, the k-means clustering algorithm can eliminate the external noise interference more effectively and divide the flame area more accurately. In the case of less external interference sources, the accuracy of the flame segmentation of 94 or more.

The experimental samples of this paper mainly from the National Forest Fire Network, Baidu, Google Photo Gallery and so on. A total of 100 samples of forest fireworks were collected and 50 negative samples were collected.

\section{CONCLUSION}

In this paper, we propose a flame segmentation algorithm based on the combination of color feature and K-means clustering of forest flame image. The K-means clustering of the forest flame image is initialized $\mathrm{k}$ to 8 and is converted to $\mathrm{YCrCb}$ color space. By separating the color channels, the gray value of the forest flame area in different color space chromaticity channels is analyzed in the sample image. The gray value of the flame on the chromaticity channel is analyzed, and the threshold of the forest flame is set to judge the image Whether there is a flame. This algorithm greatly improves the accuracy of forest flame recognition, reduces the rate of misjudgment and the rate of failure, so that it can meet the accuracy and real - time of forest fire identification. Through a large number of experiments, based on the forest flame image color characteristics and Kmeans clustering algorithm can quickly and accurately segment the flame image, and achieved good recognition results.

\section{References:}

[1]YAN Hou, NI Jin-sheng.Study on Automatic Fire Detection Technology of Forest Fire Based on Network Camera [J]. Forest Fire Prevention, 2007, (02): 25-28.

[2]Zhu Sisi, Ding Dehong, Chen Zhaoying, Zhao Fangzhen.Study on Forest Fire Recognition Method Based on Image Processing [J]. Infrared Technology, 2016, (05): 440-446. [3]Liang Jie, Zhang Lihong, Li Lin. Fire image segmentation method combining HSI and regional growth [J] .Computer Technology \& Development, 2012, (01): 191-194.

WANG Yu-bin.Study on fire smoke detection technology based on image type [J]. Fire Science and Technology, 2014,33 (9): 1052-1055.

[4] Yu Ronghua. Forest fire image automatic identification system research and implementation [D] .Nanchang University, 2008.

[5]Li Dandan, Shi Xiuzhang. Color image segmentation algorithm based on HSI space and K-means method [J]. Microelectronics and Computer, 2010, (07): 121-124.

[6] Luo Yuanyuan. Research on forest fire detection technology based on $\mathrm{YCbCr}$ color space [D]. Journal of Central South University of Forestry and Technology, 2013.

[7] $\mathrm{Hu}$ Chuanxiu.Study on Flame and Smoke Recognition Method Based on Fusion Feature [D]. East China Jiaotong University, 2016. 